\title{
Monitoring Coumadin-The Original Oral Anticoagulant
}

\section{DAVID L. MCGLASSON}

\section{LEARNING OBJECTIVES}

1. Discuss the use of the prothrombin time (PT)/international normalized ratio (INR) for monitoring subjects on the oral anticoagulant Coumadin.

2. Be aware of the relevance of clinical and genetic data (CYP2C9, VKORC1) in predicting Coumadin dosing in subjects requiring different INR ranges.

3. Be knowledgeable of the chromogenic factor $\mathrm{X}$ (CFX) assay for monitoring Coumadin in subjects with lupus anticoagulants, transitioning subjects to Coumadin from direct thrombin inhibitors and for accurately following patients with elevated INRs.

ABBREVIATIONS: CFX - chromogenic factor X; CLSI - Clinical and Laboratory Standards Institute; CYP2C9 - cytochrome P450; DTI - direct thrombin inhibitor; FII - coagulation factor II (prothrombin); FX - coagulation factor X; GMNPT - geometric mean normal prothrombin time; INR - international normalized ratio; IRP - international reference preparation; ISI - international sensitivity index; LA - lupus anticoagulant; OAT - oral anticoagulation therapy; PGX - pharmacogenetics; PST - patient self-testing; PT - prothrombin time; TTR - time in therapeutic range; VKORC1 - vitamin K epoxide reductase complex subunit 1; WHO - World Health Organization

INDEX TERMS: Coumadin, international normalized ratio, genetic data, chromogenic factor $\mathrm{X}$

Clin Lab Sci 2013;26(1):43

David L. McGlasson MS, MLS(ASCP) ${ }^{C M}$, 59 $9^{\text {th }}$ Clinical Research Division Laboratory Services, Wilford Hall Ambulatory Service Center, Lackland AFB, TX

Address for Correspondence- David L. McGlasson MS,
MLS(ASCP $)^{C M}$, Clinical Research Scientist, $59^{\text {th }}$ Clinical Research Division Laboratory Services, Wilford Hall Ambulatory Service Center, Lackland AFB, TX 782369908,210-292-6555, David.mcglasson@us.af.mil

The prothrombin time (PT) has been the primary screening test for the laboratory evaluation of patients with an acquired or inherited coagulation factor deficiency of what was originally known as the extrinsic or common pathway of coagulation. It is used in conjunction with the international normalized ratio (INR) to monitor oral anticoagulant therapy (OAT) subjects on the oral anticoagulant, Coumadin. ${ }^{1}$

Tissue factor (TF) in vivo activates the coagulation cascade through the formation of the TF/FVIIa complex. The PT adds a TF/calcium mixture to citrated platelet-poor-plasma (PPP) in vitro to obtain the time for clot formation. There are many variables such as proper specimen collection, multiple reagent/instrument combinations and, most importantly, the sources of thromboplastin, usually rabbit and recombinant tissue factor, selected for the assay. ${ }^{1,2}$

\section{The INR and Local INR Calibration}

The commercial thromboplastins vary widely in their sensitivities to Coumadin. Instrumentation differences (photo-optic or mechanical clot detection) may also play a clinically significant role in the performance of the PT/INR assay. With so many variables, providers treating subjects on OAT can be confused when comparing patient results from a number of laboratories using different reagent/instrument combinations. A facility that uses a high sensitivity thromboplastin will generate longer PT results than an assay that uses a low sensitivity thromboplastin. Therefore a subject on OAT may have a PT of 14 seconds with a low sensitivity reagent or 18 seconds with a more sensitive thromboplastin. Hence a subject monitored with insensitive thromboplastins would appear to require a higher dosage of Coumadin to result in an appropriate 
prothrombin time ratio. ${ }^{1,2}$

In 1977, the World Health Organization (WHO) realized the problems associated with comparing PTs that are performed with different reagents and introduced a standardized thromboplastin that became their international reference preparation (IRP). ${ }^{2}$ In 1983 the WHO described a model for standardizing the PT based on a method in which the PT value is reported as an INR. The INR is the PT that would be obtained if the assay were performed using a WHO primary reference with an international sensitivity index (ISI) value of 1.0. The ISI compares the sensitivity of a known thromboplastin to an international reference plasma calibrated by the manufacturer using the WHO reference plasma. The ISI is an indicator of the thromboplastin reagent's sensitivity to factor deficiencies in comparison to a known standard. The patient's PT, the geometric mean PT of the reference interval and ISI are required to calculate the INR. The outcome of this calculation is used to treat subjects on Coumadin to prevent thrombosis. ${ }^{3,4}$

The formula for calculating the INR is as follows: ${ }^{5}$

$$
\text { INR }=\left(\frac{\text { Patient PT (sec) }}{\operatorname{MN~PT~(sec)~}}\right)^{\text {ISI }}
$$

The INR compensates for differing reagent and instrument combinations. Even with different ISI values, the patient values can be theoretically compared using the calculated INR thus allowing a better and more normalized regulation of OAT.

Over the years those reviewing the INR computation have examined the assignments of the ISI values, instrument methods, and calculation errors. A wrong assignment of the ISI may result in deadly inaccurate values. These differences could result in inappropriate dosing of patients on OAT leading to bleeding or repeat thrombosis. ${ }^{2,6-8}$

INR variables have led to recommendations to locally calibrate the ISI with each laboratory reagent and instrument combination using commercial INR calibrators. A recent publication discusses local ISI validation and calibration. ${ }^{4}$ The author uses FDAcleared kits from vendors who furnish certified plasmas, techniques and examples of data calculations. The procedure can be performed in a laboratory that currently performs a PT/INR. The validation requires three days of testing on certified plasmas (INRs from 1.5-4.5) and calculations that are performed locally or using an automated vendor template. This local calibration should be performed any time a new reagent/instrument or lot of reagents is changed for performing a PT/INR assay in the facility. Each laboratory should be responsible for determining the local ISI, as the manufacturer cannot duplicate the performance of the local test set-up or the proficiency of the employees performing the testing for OAT. The necessity for local calibration of the ISI is imperative and should be performed following strict guidelines from the Clinical and Laboratory Standards Institute (CLSI). ${ }^{4,5}$

By reporting a locally calibrated INR, each facility is using a standardized unit related to the WHO standards. The INR provides the opportunity to use a common unit for defining OAT therapeutic ranges. Clinicians treating subjects being monitored for OAT for arterial or venous thromboembolism target an INR of 2.0-3.0. The target range for a patient with a mechanical heart valve may be $2.5-3.5 .^{9}$

\section{Monitoring Coumadin Using POC Instrumentation} Point of care (POC) instruments are available that are able to determine patients' INR from capillary blood. POC reagent cartridges contain only thromboplastin since the specimen is not citrated blood. These instruments are primarily used in anticoagulation clinics, physician offices, or by OAT patients using home-care devices. However, there are no INR calibrators available to locally calibrate the ISI on POC devices and they should be compared to the main facility's reference method when confirming critical values.

OAT efficacy is influenced by the patient's diet, supplement interactions, body mass index, age, gender and liver functions. The INR should be carefully followed with regard to the subject's drug regimen, wellness and dietary changes. ${ }^{10}$ An INR result is not meaningful in subjects who are not on OAT.

When to test and time in therapeutic range (TTR) of 2.0-3.0 are extremely important to prevent the risks of a bleeding event or stroke. Dr. Jack Ansell, MD, international anticoagulation expert and the driving 
force behind the Anticoagulation Forum recently presented the results of the Self-Testing Analysis Based on Long-Term Experience (STABLE) study at the March 2012 American College of Cardiology Summit. This study looked at 29,500 U.S. based, real-world patient self-testers (PST) for more than two years and found that PST using POC testing as part of a comprehensive support service had a significantly higher percentage of TTR $(74.0 \%)$ when they tested weekly. They also benefited from reductions in time spent in the critical INR ranges (INR $<1.5$ or $>5.0$ ), which may triple prevalence of stroke or major bleeding events, respectively. This study states, "by applying more frequent self testing to warfarin therapy, one can optimize safety and efficacy." The TTR demonstrated in this study surpassed that of other, well-designed clinical trials, which included the 2,922 patient, VA Cooperative THINRS trial (TTR $=66.2 \%)$, presented at American Heart Association meeting in 2008. These data come just before the American College of Chest Physicians (ACCP) released clinical evidence in support of patient POC testing over usual outpatient INR testing. ${ }^{11,12}$

Conversely, another study of 250 subjects monitored over a year evaluated Coumadin dose assessment every 4 weeks versus every 12 weeks in patients with stable INR. ${ }^{13}$ They investigated whether assessment of warfarin dosing every 12 weeks was as safe as every 4 weeks. They monitored subjects whose dosing was unchanged for at least six months. The TTR was $74.1 \%$ in the 4-week group compared with $71.6 \%$ in the 12 week group. The authors' conclusion was that assessment of warfarin dosing every 12 weeks seems to be as safe as testing every 4 weeks. The testing was performed in a single anticoagulation clinic in which participants coordinated with clinic personnel every four weeks. ${ }^{13}$ This is a smaller study than previously discussed but it demonstrates how a well-controlled patient population can be maintained in the TTR with fewer testing times. Even though the data conflict with the previous study it seems that monitoring patients frequently through special anticoagulation clinics seems to keep individuals in the TTR better than a single physician setting.

\section{Screening for Mutations Prior to Coumadin Therapy} Genetic testing for OAT was discussed starting in the early 2000's. ${ }^{14}$ The FDA even commented on the subject (Critical Path Initiative: Warfarin dosing on
July 27, 2007, www.fda.gov/oc/initiatives/criticalpath/ warfarin.html) stating that patients would greatly benefit from genetic testing to enable warfarin dosing. ${ }^{15}$ However, as yet this is not a frequently-requested test.

OAT dosing is difficult for a number of reasons. The narrow therapeutic window and wide range of individual response can be influenced by age, gender, diet, medication interactions, disease state, and variations in genetic polymorphisms. ${ }^{16}$ Differences in a gene of the cytochrome oxidase-reductase system (CYP) $\mathrm{P} 450,2 \mathrm{C} 9$ gene CYPC2C19 and in the vitamin $\mathrm{K}$ epoxide reductase (VKOR) $\mathrm{C} 1$ (VKORC1) are associated with slowed Coumadin metabolism. A subject's ability to respond avidly to Coumadin is controlled by the VKORC1 gene. ${ }^{14}$ This gene controls the site of action where vitamin $\mathrm{K}$ is reduced. Subjects who have the more sensitive genotype usually require a lower dose than the average patient. This can be referred to the AA genotype. Those who are resistant to the effect of Coumadin usually carry the GG genotype and require stronger dosing. Conversely, the CYP2C19 gene is responsible for metabolizing active Coumadin. ${ }^{14}$ Subjects who possess CYP2C19 variations metabolize Coumadin more slowly than subjects who don't. These patients take longer for the INR to reach a TTR and may require a lower dose of Coumadin than someone who metabolizes warfarin faster.

An article in the May, 2012 CAP Today discusses how testing for Warfarin ${ }^{\oplus}$ pharmacogenomics (PGX) is still a waiting game. No professional groups have endorsed genetic testing as a standard assay to enable Coumadin dosing. Cost is upwards of $\$ 500.00$ in most reference labs. There are only two vendors who currently provide materials and a platform for Coumadin PGX analysis. In August of 2009, the Centers for Medicare and Medicaid Services decided not to reimburse testing as part of routine patient care. Nevertheless, the FDA again the following year recommended genotyping prior to initial dosing. Dr. Charles Eby at Washington University in St. Louis, states "For many, many, many physicians, it's a novelty that is not part of their practice tradition." Dr. Eby saw the FDA's statement as a positive step because the agency said it would pay for the testing if it was part of a clinical trial. In the real world, the turn-around-time of PGX testing can be as long as 30 days. Few physicians can wait that long to treat a subject with Coumadin when they need to be 
anticoagulated. The controversy continues on the use of PGX for anticoagulating with OAT. For more information about Coumadin monitoring and dosing recommendation algorithms go to www. WarfarinDosing.org. The site's algorithm has been validated and used on more than 1000 patients. ${ }^{17}$

\section{The Chromogenic Factor X Assay Monitors Coumadin}

We have discussed how the INR is the principle method for monitoring patients on OAT. However there are many preanalytical variables that can affect the INR, for instance, the presence of a lupus anticoagulant (LA). This is especially true when the reagent and instrument combination of the test system has not been locally calibrated for the ISI. An alternative to the INR in the case of interference is the chromogenic factor X (CFX) assay. The CFX has been shown to be insensitive to many of the variables such as LA that may affect the INR. ${ }^{18-22}$

A number of researchers have established the CFX therapeutic range in the presence of an LA that corresponds to the therapeutic range of the INR. ${ }^{23-24}$ Further, McGlasson (2008) postulated that the INR might be invalid due to the range "flattening out" when the INR result is $>3.0$. This data showed it was possible to have a subject with a very high critical value of INR and still have a CFX level in the therapeutic range. ${ }^{23}$ This article suggests that the INR be replaced by the CFX in monitoring all subjects on Coumadin, especially for individuals with INR values $>3.0$ (Figure $1)$.

Rosborough et al showed how FII and FX activity levels did not always agree in Coumadin-treated LA subjects. ${ }^{25}$ Their data demonstrated that the CFX method was preferred to the INR and clottable based factor assay in monitoring subjects with an LA who were on OAT. They also established that the relationship of CFX to INR testing differs during Coumadin initiation compared to during chronic Coumadin therapy. ${ }^{26}$ Their conclusion was that this would have implications for warfarin dosage protocols in patients requiring CFX monitoring. Rosborough also described the relationship between the INR and CFX in the samples from patients in their study compared to the reciprocal transformation of the INR seen by the McGlasson study. They noted the problem with the INR when the value was $>3.0$ (see Figure 2). ${ }^{25}$

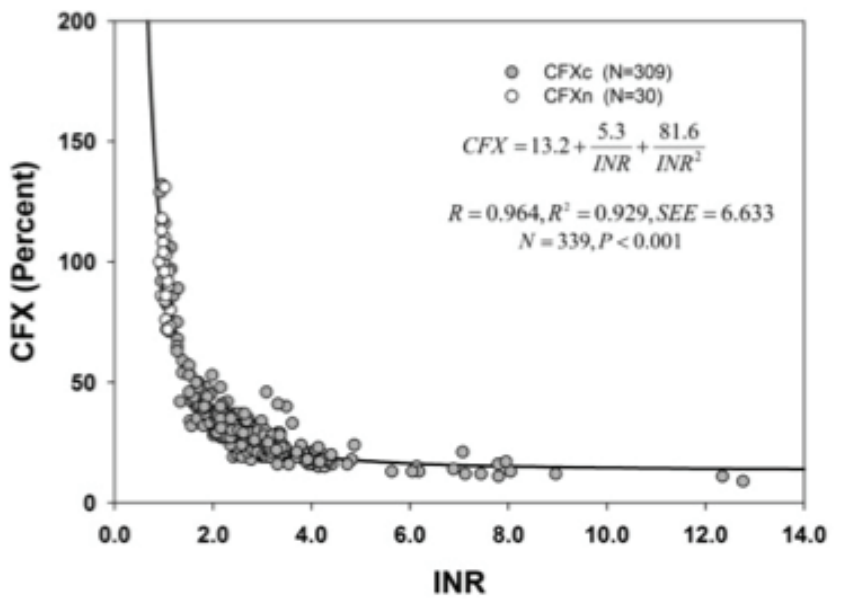

Figure 1. McGlasson DL, Romick BG Rubal BJ. Comparison of a chromogenic factor $\mathrm{X}$ assay with international normalized ratio for monitoring oral anticoagulation therapy. Blood Coagul Fibrinolysis 2008;19:513-7. Published with permission.

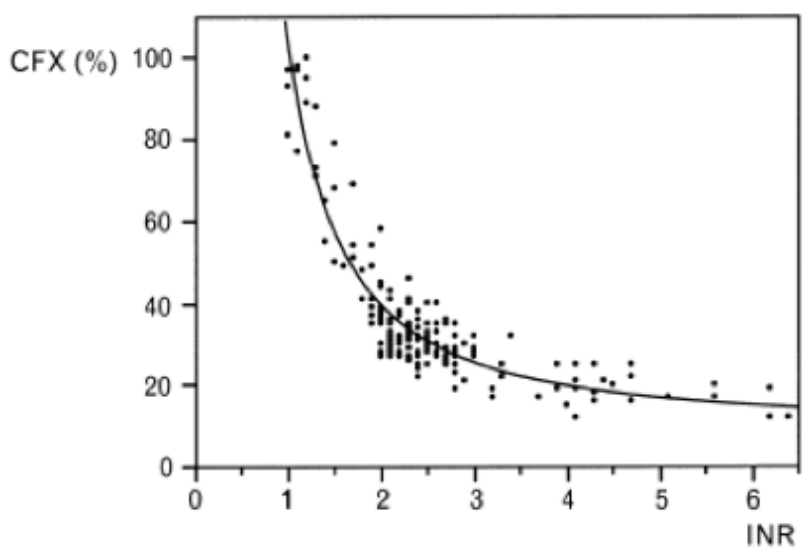

Figure 2. Rosborough TK, Jacobsen JM, Shepherd M. Factor X and factor II activity levels do not always agree in warfarintreated lupus anticoagulant patients. Blood Coagul and Fibrinolysis 2010;21:242-4. Published with permission.

When monitoring subjects on OAT there are many preanalytical variables that can affect the outcome of INR testing. Frequent testing in conjunction with carefully monitored anticoagulation clinics may give the patient a better chance of staying within the therapeutic range, thus enhancing their opportunity to remain incident free.

\section{REFERENCES:}

1. Hirsch J, Poller L. The international normalized ratio-a guide to understanding and correcting its problems. Arch Intern Med 1994;154:282-8.

2. McGlasson DL. A comparison of INRs after local calibration 


\section{FOCUS-ANTICOAGULANT THERAPY}

of thromboplastin International Sensitivity Indexes. Clin Lab Sci 2003; 15:91-5.

3. Poller, L. International normalized ratios (INR): The first 20 years. J Thromb Haemost 2004;2:849-60.

4. Marlar RA, Gausman JN. Do you report an accurate international normalized ratio? Find out using local verification and calibration. Lab Medicine 2011;42:176-81.

5. CLSI. One stage prothrombin time (PT) test and activated partial thromboplastin time (APTT) test: Approved guideline. H47A2. Wayne, PA: CLSI:2008.

6. Adverse events and deaths associated with laboratory errors at a hospital-Pennsylvania 2001. MMWR 2001:710-11.

7. McCullough M. Lab error deaths may now total five. The Inquirer (Philadelphia, PA), August 3, 2001.

8. McGlasson DL. Laboratory variables that may affect test results in prothrombin time (PT)/International normalized ratios I(NR). Lab Medicine 2003;34:124-8.

9. Ageno $W$ et al: Oral anticoagulant therapy: antithrombotic therapy and prevention of thrombosis, $9^{\text {th }}$ ed: American College of Chest Physicians evidence-bases clinical practice guideline. Chest 2012;141(2_suppl) e44S-88S.

10. Budnitz DS, Pollock DA, Weidenbach KN et al. National surveillance of emergency department visits for outpatient adverse drug events. JAMA 2006;296:1858-66.

11. www.businesswire.com Largest warfarin patient self-testing study results establish new standard of care. Accessed 09.12.2012.

12. www.ptinr.com/warfarin.Alere's STABLE study redefines warfarin safety and practice management. Accessed 09.12.2012.

13. Schulman S, Sameer P, Stewart MA et al: Warfarin dose assessment every 4 weeks versus every 12 weeks in patients with stable international normalized ratios: A randomized trial. Ann Intern Med 2011;155:653-9.

14. Bussey HL, Wittkowsky AK, Hylek EM, Walker MB. Genetic testing for warfarin (Coumadin) dosing?-Not yet ready for prime time. Accessed www.clotcare.com/warfaringeneticetest ing.aspx 09.12.12

15. Critical Path Initiative: Warfarin dosing 27 July 2007. Accessed 09.12.12. www.fda.gov/oc/initiatives/criticalpath/war farin.html
16. Gage BR, Eby C, Milligan PE et al: Use of pharmacogenetics and clinical factors to predict the maintenance does of warfarin. Thromb Haemost 2004;91:87-94.

17. Titus K. Warfarin pharmacogenetics a waiting game. May 2012 CAP Today

18. McGlasson DL, Shaklee PN. A multi-instrument evaluation of a commercial chromogenic factor $\mathrm{X}$ assay to monitor oral anticoagulation therapy. Blood 2004;104:4059abs.

19. Ortel TL, Moss S. Monitoring oral anticoagulant therapy in patients with lupus anticoagulants (comment). Br J Haematol 1998;101:390-2.

20. Robert A, LeQuerrec A, Delahousse B et al: Control of oral anticoagulation in patients with the antiphospholipid syndrome: influence of the lupus anticoagulant on international normalized ratio. Groupe Methodologie en Hemostasise du Groupe d'Etudes sur l'Hemostasis et la Thrombose. Thromb Haemost 1998;80:99-103.

21. Rosborough TK, Shepherd MF. Unreliability of international normalized ratio for monitoring warfarin therapy in patients with lupus anticoagulant. Pharmacotherapy 2004;24:838-42.

22. Balaban M, Stanric V, Rincic G et al: Recurrent venous thrombosis despite optimal anticoagulation therapy for antiphospholipid syndrome-could new oral anticoagulants solve the problem? [Review]. Acta Clinica Croatia 2012 49:469-77.

23. Moll S, Ortel TL. Monitoring warfarin therapy in patients with lupus anticoagulants Ann Intern Med 1997;127:177-85.

24. McGlasson DL, Romick BG Rubal BJ. Comparison of a chromogenic factor $\mathrm{X}$ assay with international normalized ratio for monitoring oral anticoagulation therapy. Blood Coagul Fibrinolysis 2008;19:513-7.

25. Rosborough TK, Jacobsen JM, Shepherd M. Factor X and factor II activity levels do not always agree in warfarin-treated lupus anticoagulant patients. Blood Coagul Fibrinolysis 2010;21:242-4.

26. Rosborough TK, Jacobsen JM, Shepherd MF. Relationship between chromogenic factor $\mathrm{X}$ and international normalized ratio differs during early warfarin initiation compared with chromic warfarin administration. Blood Coagul Fibrinolysis 2009;20:433-5. 